\title{
INPUT SOURCES AND PROPERTIES OF POSITION- SENSITIVE OCULOMOTOR FIBRES IN THE ROCK LOBSTER, PANULIRUS INTERRUPTUS (RANDALL)
}

\author{
By B. YORK,* K. YANAGISAWA $\dagger$ AND C. A. G. WIERSMA \\ Division of Biology, California Institute of Technology \\ Pasadena, California, U.S.A.
}

(Received II Fanuary 1972)

\section{INTRODUCTION}

The positions of the eyecups of stalk-eyed decapod Crustacea are mainly determined by several sets of muscles which influence the angle at which the eyes are kept with regard to the proximal joint of the eyestalk. As the animal's position changes this angle also changes in such a way as to keep the eyes in a horizontal plane, and as near as possible to their former position. In the crayfish the muscles responsible consist of two sets of antagonistic pairs, which have been called the eye-up and eye-down and the head-up and head-down muscles, according to the body position which excites their motor axons (Wiersma \& Oberjat, 1968). In crabs the situation is more complex in that more than two pairs are involved (Burrows \& Horridge, 1968). It will be shown that the rock lobster resembles the crayfish, having also but two sets of mutually antagonistic position-sensitive motor fibres.

The eyecup can be moved to a retracted position by a large muscle which, in the crayfish, moves the eye forward or, in many crabs, backwards. This protective withdrawal movement in the rock lobster, which consists of a much smaller relative motion, will be shown to be governed again by a separate nerve-muscle system.

In Carcinus no visual input is found for any but the horizontal optokinetic motor fibres (Wiersma \& Fiore, 197I $b$ ), whereas in the crayfish position-sensitive fibres do respond to visual stimuli (Wiersma \& Oberjat, 1968). It will be shown that the rock lobster also resembles the crayfish in this respect. Other influences on the discharge rates of the position fibres, such as those relating to the positions of the legs and head appendages, will also be described and discussed.

\section{METHODS}

The recording methods used have been presented in the preceding paper. To determine which type of motor fibre was giving the signals, the animal was placed in various body positions by manipulation of its clamp. In general this was done by holding the clamp by hand. Eye-up fibres will discharge most when the eye is up and least when it is down, and similarly all fibres have been named for the position

\footnotetext{
- Present address: Department of Physiology and Biochemistry, University of Southampton, England.

+ Present address: Department of Physiology, Tsurumi Women's University, Yokohama, Japan.
} 
causing their maximal discharge. Very few experiments have been carried out in which mechanical rotation was continuous so as to relate the body position and the accompanying discharge rate, because the heavy weight of the preparation made such experiments difficult. Instead, the animals were clamped in a few stationary positions to indicate the relationship between body angle and firing frequency. For investigation of the influence of moving stripes, the animal was variously mounted within the drum as mentioned in the Results. Manipulation of appendages was mainly by hand.

\section{RESULTS}

\section{General}

No more than three fibres of any one type were recorded in one lead. As for the optokinetic motor fibres described in the preceding paper (York, Wiersma \& Yanagisawa, 1972), all fibres of one type respond similarly to all the same stimuli but with different thresholds and firing rates. Again the threshold increases with fibre size. The position-sensitive fibres are stimulated by an excited state, as in the crayfish (Wiersma \& Oberjat, I968) and in the rock lobster's optokinetic fibres. As in other species there is no indication of joint receptors at the level of the outer eyestalk. In a considerable number of experiments we investigated whether more than two sets of position fibres could be found, as in the crab (Burrows \& Horridge, 1968; Wiersma \& Fiore, 197 $a, b$ ), but none were encountered. Nor was there any evidence for the presence of unidirectional motor fibres though the phasic fibres of each set, because of their preference for rotation from the normal to the maximally effective position, showed under habituated conditions a discharge type which could be mistaken for unidirectionality. The eye-up and eye-down, the head-up and headdown and, in addition, the eye-withdrawal fibres will now be considered separately.

\section{Influence of body positions}

\section{Eye-up and eye-down fibres}

We will report here mainly on the reactions of these fibres to inputs other than from the statocysts for the reasons mentioned in the Methods. But when gradual rotation of the animal around its longitudinal axis is performed in darkness it causes a gradual increase in discharge frequency until the approximate maximally effective position is reached; however, this sustained firing rate shows, as in other species, a quicker increase when the position is reached from the normal, rather than from the upside-down, position (hysteresis). Though this body-position effect is usually the strongest input, we found that in a few animals the influence of the location of a light source was stronger. In such cases it was necessary for determination of the nature of the fibre to perform the position changes in darkness, as otherwise the light effects described below could override the body-position effects and lead to wrong conclusions.

\section{Influence of visual stimuli}

Two types of visual input are present, namely optokinetic and light direction. For investigating optokinetic influences the animal was mounted within a striped drum, usually in the head-down but sometimes in the head-up position, so that the vertical 

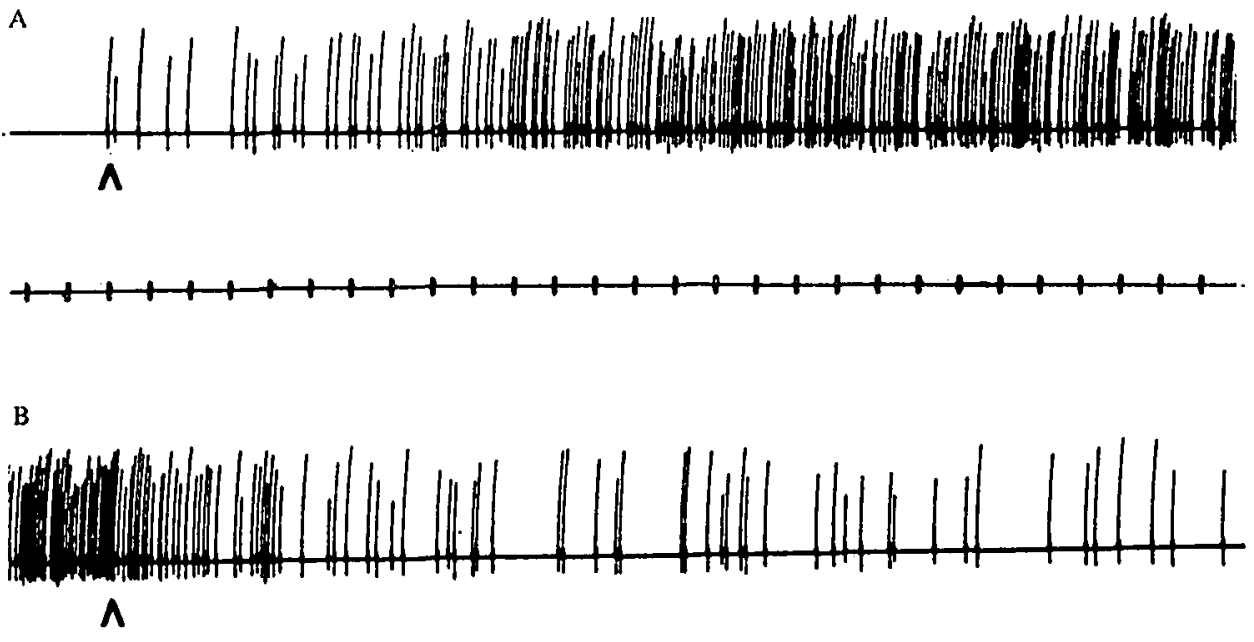

Text-fig. 1. Optokinetic input on to a tonic eye-up fibre; animal mounted head-downward in drum with vertical stripes. (a) Excitation due to rotation in the preferred direction (dorsal to ventral). (b) Inhibition due to rotation in the unpreferred direction (ventral to dorsal). Arrows denote start of rotation at $3 \%$ sec. Time: 1 sec.

stripes would travel over the eye surface in either direction with a large component in the vertical axis of the eye. (Because of the reaction of the head-down, head-up fibres, respectively, the eye axis is not in the vertical plane when the body is.) In these positions the background frequency is similar to that in the normal body position. In about one-third of the animals no optokinetic influence could be found in these fibres. When present it was usually weak and habituating. However, it could occasionally be of considerable magnitude (Text-fig. I). Rotating the animal is, in general, more effective than rotating the drum at the same speed though, as in the optokinetic fibres, rotation of the animal in the dark does not result in a significant change in discharge. By blocking the vision of one eye or the other with a dark cap it was found that some animals showed binocular input while in others only the ipsilateral eye was involved. When bilateral input was present, the major contribution was almost invariably from the ipsilateral eye. As in the optokinetic fibres (see preceding paper) the frequency build-up depends on drum speed, and on stopping the drum during rotation in the preferred direction afterdischarge is sometimes evident.

More pronounced is the influence due to illumination of the eyes. For eye-down fibres, light shone anywhere in the ipsilateral eye is inhibitory and in the contralateral eye excitatory. For eye-up fibres the reverse is true. In several preparations light was more effective when shone in certain areas of the eye than in others, both with regard to excitation and inhibition. In about $20 \%$ of the animals light input was ineffective, but otherwise it was usually binocular. Only in one case was the contralateral, but not the ipsilateral, effect present. The presence of the light effect is not necessarily accompanied by optokinetic input. When a fibre reacted to light, fast shadowing (by moving an object to and fro before the eye under room-light conditions) affected the frequency distribution of the impulses, and phase-locked impulses for shadowing frequencies of up to ro/sec. despite the small intensity changes involved (see Pl. I, 
fig. 4 for the same phenomenon in a head-down fibre). Remarkably enough this effect was always limited to the ipsilateral eye. Therefore, in eye-up fibres the excitatory input is affected, whereas in eye-down fibres the inhibitory input is affected.

\section{Antennule positions}

The effect of changing the antennule position was investigated in several preparations. Theoretically this can be quite a complex stimulus. Not only will the position of the statocysts, located at the base of the antennule, obviously be changed, but floating hairs in the statocyst may be activated by fluid movements. Even less controllable, joint receptors such as those described for the antennules of the related species Palinurus vulgaris (Schöne \& Schöne, 1967) may enter the picture. Not unexpectedly the most obvious reaction in the eye-up and eye-down fibres occurs on rotating the two antennules simultaneously around their long axes either clockwise or anticlockwise, since the statocysts will thus be rotated relative to the gravitational field. Turning the statocyst opening of the ipsilateral antennule towards the eyecup excites the eye-down fibre (Pl. I, fig. I) and inhibits the eye-up fibre. The frequency changes obtained were not as large as when the whole animal was rotated. More unexpected was the regular finding that bringing the antennules towards the ipsilateral eye in the horizontal plane (thus excluding gravity as a variable) resulted in an increase in firing rate in the eye-up fibres and a decrease in the eye-down ones, whereas movements in the opposite direction had the reverse effect (see Text-fig. 2). These influences outlasted the antennule movement. Large eye movements in the appropriate up-and-down directions occurred simultaneously.

\section{Antennae positions}

Antennal influences were also present although these were much weaker than those of the antennules. The large number of antennal joints makes it somewhat difficult to decide how far each contributes to the input. For an eye-down fibre, bringing the ipsilateral antenna base backward and the flagellum upward excited, whereas bringing the base forward and flagellum downward inhibited (Pl. I, fig. 2). Any effect of the contralateral antenna was doubtful. As will be discussed later, ipsilateral antennal input is probably related to the pointing reaction.

\section{Leg positions}

In confirmation of the experiments on eye movements by Dijkgraaf (1956), a strong influence of leg position on the firing rates of these fibres was found. The input was of the push-pull type. For the homolateral eye-down fibre, flexing the ipsilateral legs and extending the contralateral legs excited, whereas movements in the opposite directions were inhibitory ( $\mathrm{Pl}$. I, fig. 3). Each leg provided a noticeable influence when manipulated singly. Included were all appendages from the third maxilliped to the fifth leg. Input was especially from the basi-ischiopodite joint. This reaction always occurred despite the presence of a reflex which causes the antennal flagellum to be lowered when the legs are flexed. As stated, such antennal movement by itself results in an inhibition of the eye-down fibres. This indicates that the leg input overrides the antennal one, though it is not excluded that the antennal influence might be different depending on whether or not its motion is active. That the antennal 


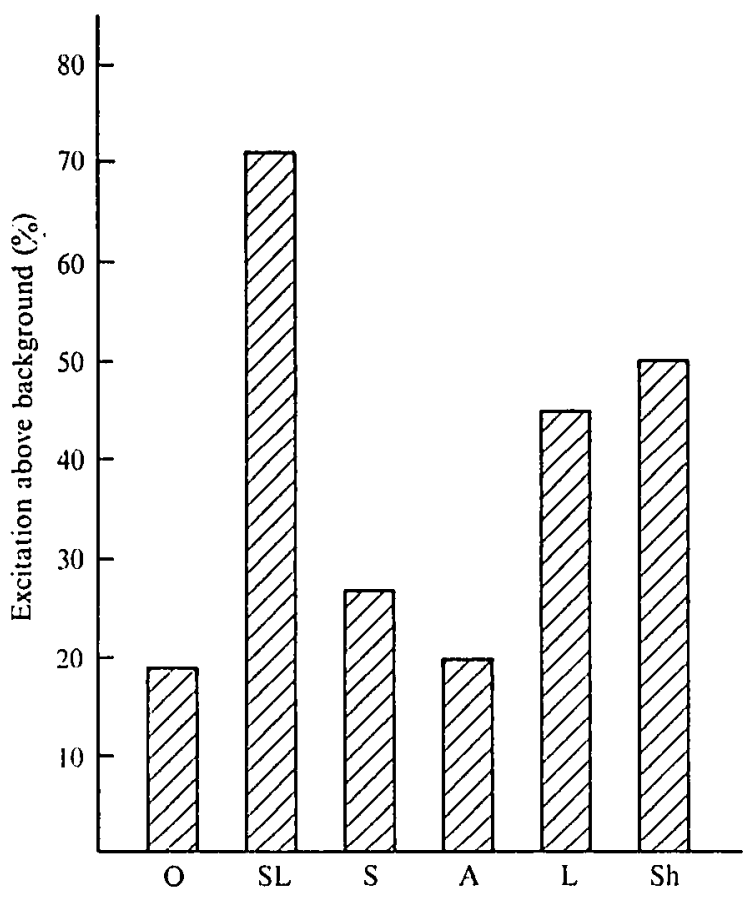

Text-fig. 2. Relative excitatory inputs from body position, light, antennule position and shadowing on a tonic eye-down fibre. Input strength is expressed as percentage increase in firing rate above background.

O: optokinetic input from drum rotation in the preferred direction; SL: statocyst and light input from rotating the animal eye-down in the light (4 foot-candles); $\mathrm{S}$ : statocyst input from rotating the animal eye-down in the dark (leg-position unchanged); A: antennule movement input on bringing both antennules away from the ipsilateral eye in the horizontal plane; L: leg-joint input on flexing the ipsi- and extending the contralateral legs; Sh: input from shadowing the ipsilateral eye (light-intensity level was 4 foot-candles).

input is indeed weaker than the leg input is strengthened by the observation that only the most tonic fibre reacted to it, whereas the more phasic ones also reacted to leg manipulation. The efficiency of the leg-joint input is further indicated by the fact that it can override the combined inhibitory influences of light and statocyst in the eye-up position on an eye-down fibre.

Text-fig. 2 shows the relative effectiveness of most of the inputs so far described on one particular eye-down fibre. The relative effectiveness of these inputs on any one type of fibre varied remarkably from animal to animal. For example, in this preparation the light input was strong compared to the statocyst input, but this is by no means always true.

\section{Head-up and head-down fibres}

Only a few head-up fibres were studied, but with regard to the experiments to be described their overall reactivity was much like that of the head-down fibres. Of course, they differ from each other in that the effects of all inputs except for the excited state were of the opposite sign.

Optokinetic input to these fibres was found to be present, but was difficult to investigate for technical reasons. The stimulus used was a white half-globe, with 


\section{4 \\ B. York, K. Yanagisawa and C. A. G. Wiersma}
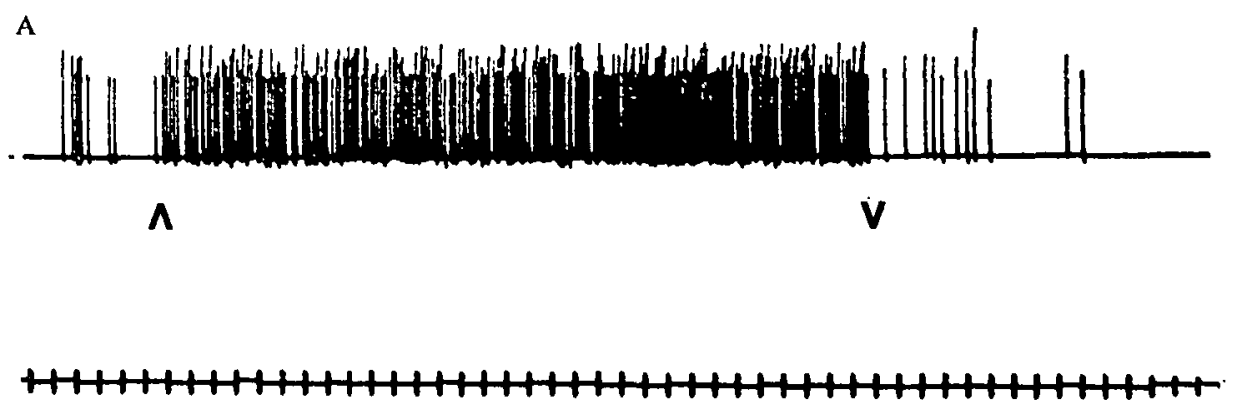

B

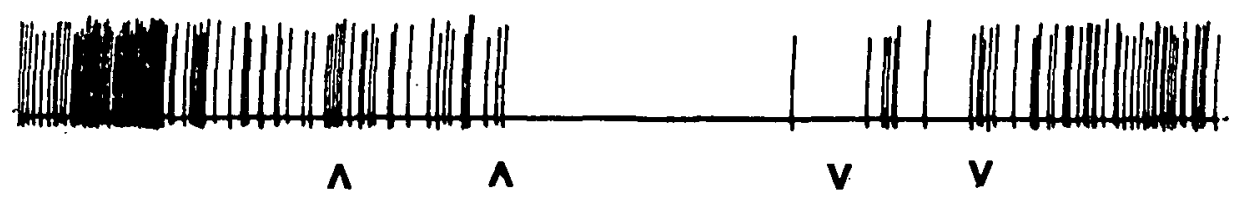

Text-fig. 3. Opposing effects of antennule movement in the vertical plane on two head-down fibres from two different animals. (a) Lifting both antennules excited the fibre (Ist arrow); dropping (2nd arrow) and subsequently depressing them, inhibited. (b) Lifting first the contralateral (Ist arrow) and then, in addition, the ipsilateral (2nd arrow) antennule inhibited the fibre; depressing first the contralateral (3rd arrow) and then, in addition, the ipsilateral (4th arrow) antennule excited. Time: I sec.

8 black stripes arranged on the inside as spokes, which was rotated around the eye's axis in front of the eye. It had the same but much weaker effect in comparison to rotation of the whole animal in the opposite direction around the same axis.

The discharge rate of the head-down fibres usually increases when light is shone in the back rim area (that is, in the field of the back rim sustaining fibre LO 27), whereas light in the front rim inhibits. Out of 9 tested preparations, 6 showed good bilateral sensitivity, I ipsilateral sensitivity only, whereas 3 showed no significant light influences. In cases with bilateral input, shadowing was effective over the ipsilateral eye only, as in eye-up and eye-down fibres (Pl. I, fig. 6).

For 7 head-down fibres, pulling the antennules upward inhibited the fibres, and pulling them downward excited. However, in two fibres exactly the opposite influences were found (Text-fig. 3). This may indicate that beside the statocystposition input, the stretch receptors described by Schöne come into play, the two inputs having opposing effects. In a number of animals kept in the storage tank the eyes were normally found to move upward on bringing the antennules down when the animal was held horizontal, indicating that the head-down fibres were stimulated, as in the case of the above-mentioned 7 instances.

Leg positions can have a weak influence on the discharge rate. Occasionally, bringing all legs backward resulted in a decreased discharge rate in the head-down fibre, while a slight increase occurred on bringing them forward. The coxo-basipodite joint appears to be involved. Some weak influence of the antennal position also appears to be present, but has not been resolved, for such weak effects are difficult to distinguish from those caused by slight changes in excited state. 


\section{Eye-withdrawal fibres}

In most crabs the eye is withdrawn for protection backward into a groove in the anterior carapace region; in the crayfish a similar protective movement is by a forward motion, which hides the eye under the rostrum. In the rock lobster, neither of these two ways is present, perhaps because the normal protection of the eye is by the antenna. Instead, on stimulation of the eyecup itself, or the surrounding areas, an eye motion takes place which lowers the eye and brings it forward to about the same extent, but this movement is, in both directions, maximally less than $45^{\circ}$. It could therefore consist of simultaneous contractions of the eye-up and head-up muscles; that this is not the case is shown by the fact that both muscle action potentials and motor fibre impulses can be led off during reflex excitation, and are absent when either head-up or eye-up position is given. Therefore, this movement is equivalent to a withdrawal reflex and is executed, as in other species, by a special muscle with separate innervation. Another certain distinction is that the influence of the excited state on the discharges in the withdrawal system are the opposite from that on the other optomotor fibres, being inhibiting (Pl. I, fig. 5) and thus equivalent to those on the withdrawal reflexes of the crab and the crayfish.

The motor innervation is most probably limited to a single, rather tonically firing fibre. In all cases in which the fibre was obtained in a peripheral lead, only a single signal was obtained, and the correspondence with muscle action potentials of the withdrawal muscle was very good. In central leads, this fibre's discharges are often accompanied by those of certain other motor fibres, which are different from those so far described. They may be involved in the activation of more centrally located muscles, which attach to the proximal eyestalk, as shown for the rock lobster by Parker \& Rich (1893), but we have too few observations to state with any certainty to what stimuli these fibres react or what function their muscles have. A further study may be rewarding as some at least have been found to react to visual input.

\section{DISCUSSION}

The position-sensitive optomotor fibres in the rock lobster have a great variety of inputs, among which are those from visual stimuli, joint receptors of appendages and the statocysts. Most of these inputs give information on body position relative to gravity, and changes in body position. For example, the influence of light distribution on the eye would be an indication of the position of the sky relative to the lobster, thus reinforcing the information from the statocysts. The leg-joint input would serve as a useful indication of changes in body position. All such inputs tend to stabilize the eye position in space, and thereby the visual image on the eye, despite changes in body position, and thus increase the precision of visual reception.

A second role of the position-sensitive fibres may involve tracking of large targets, as suggested for the optokinetic fibres in the preceding paper. Movements of single objects in the vertical plane might be tracked through their optokinetic effect on the eye-up and eye-down motor fibres. Again the loose coupling between the two eyes, as suggested by the variability of the contralateral optokinetic input, could allow for independent tracking by the two eyes. As discussed later, the antennal pointing reaction may again be involved in this tracking. 
With regard to visual input, the position-sensitive fibres resemble similar fibr in the crayfish (Wiersma \& Oberjat, 1968), whereas in the crab comparable motor neurones are unresponsive to visual events (Wiersma \& Fiore, 1971 $a$ ). As in the lobster optokinetic fibres, the contralateral eye provides variable optokinetic input, ranging from ineffectiveness to clear responsiveness, to both animal rotation and drum rotation. Again, whenever there is a difference, animal rotation gives the stronger effect. With regard to illuminating the eyes, the ipsilateral effect is usually strongest, and differs from the contralateral input in that it alone shows an effect of shadowing, whether this be excitatory (eye-up fibres) or inhibitory (eye-down fibres). It should be realized that shadowing provides only for a dimming of light and is a very weak stimulus, though it is known to greatly influence the firing pattern of both sustaining and dimming fibres (Wiersma \& Yamaguchi, 1967). We tentatively ascribe the difference between ipsi- and contralateral effectiveness to the intercalation of an interneurone for the transmission of information from the contralateral eye. The passage of an extra synapse in this pathway might obliterate the bursts caused by shadowing, especially since even ipsilateral eye-shadowing, though clearly effective, is always less so than on the sustaining fibres, which form part of the input pathway.

One might ask why so many inputs, all giving information on body position, are so prominent in these fibres, rather than the statocyst input alone. This may well be connected with the fact that the proximal joint of the antennule, which carries the statocyst, is much more mobile in these animals than in other species. There are indications that the effects of antennule movement, relative to the animal's body, are at least partially compensated for by information derived from a stretch receptor at its base, as described by Schöne \& Schöne (1967) for Palinuris vulgaris, but this mechanism may not be very precise. Unlike Schöne, we found that imposed antennule movement always resulted in eye movement. However, in some animals the effect appeared to be opposite to that normally encountered. These might be instances where the stretch receptor overrode the statocyst input. The unreliability of this input from the statocysts would be greatly mitigated by strong accessory inputs indicating body position, such as those from light position on the eye and leg flexion. Also additional inputs might be very effective when either or both antennules were lost, or a statocyst was non-functional as a result of the failure to replace the statolith mass after a moult.

The influence of the input from the joints of the antennae on the position-sensitive fibres is opposite to that expected for an indication of body position. For example, in the eye-down position the antenna would tend to fall downward under its weight, yet a passive movement of the antenna in this direction causes the eye to move downward since the eye-up fibre is stimulated. This effect of the antenna on eye position would be useful, however, in the pointing reaction (Wiersma \& York, 1972) because, as in the optokinetic motor-fibre system, it orients the eye toward the direction in which the antenna points. By this means more of the eye's surface is able to react to movements of an interesting object toward which the lobster points.

The reflexes on leg extension and flexion are as expected in that they all tend to stabilize the eye's position in space during tilting of the body by leg flexion or extension of the legs. The pathway for this reflex activity for the eye-up and eye-down 
fibres may well consist of two interneurones similar to those found in the circumbesophageal connective of the crayfish. These fibres showed a push-pull arrangement with regard to input from the coxobasal joint, and were therefore named 'Sherrington's' interneurone (Wiersma, I958). The strength of these reflexes in the rock lobster, which has already been noticed by Dijkgraaf (1956), makes them a useful source for compensation for statocyst loss. However, rock lobsters climb well, and under certain conditions it would seem that the leg-position signals would be in conflict with statocyst signals. Only further experimentation can solve the problem of how these two inputs interact.

Further work is also needed to determine the interactions between all the inputs on to the motor fibres under various conditions. The factors underlying the remarkable variation in the relative effectiveness of these inputs in different preparations also pose an interesting problem. In addition, the more centrally located eye muscles and their fibres need further study for they might play a part during a movement of both eyes, which can be observed when the animal 'looks' at an object in front and above its head. In view of the fact that the eye-withdrawal motion in the lobster should make much less difference in the eyes' visual field than in other species, the inhibitory effect of the excited state on this fibre may be taken as an indication that normal eye position must be important for visual functions.

The research reported here has been supported by grants from the National Science Foundation (GB-693 I X) and Public Health Service, National Institutes of Health (NB-03627).

\section{SUMMARY}

I. Sets of head-up, head-down, eye-up and eye-down motor fibres were studied in the oculomotor nerve of the rock lobster. An eye-withdrawal fibre was also investigated.

2. Apart from the statocyst input, light distribution on the eyes has the strongest influence on the position-sensitive fibres. Weaker optokinetic input from moving targets is also present.

3. Strongly habituating input is obtained from the antennal joints. This input causes orientation of the eye toward the direction in which the antenna points.

4. The same antennule movement in the vertical plane can result in either excitation or inhibition of the head-down fibre, suggesting the presence of two opposing inputs, presumably from the statocysts and basal joint receptors of the antennule.

5. The inputs on to the position-sensitive fibres which indicate body position are such as to stabilize the eye position in space during body movement. The optokinetic and antennal joint inputs are probably involved in tracking and antennal pointing reactions.

6. The eye-withdrawal fibre is stimulated by touch of the head and around the eye, but is inhibited by the excited state. 


\section{REFERENCES}

Burrows, M. \& Horridge, G. A. (1968). The action of the eyecup muscles of the crab, Carcinus, during optokinetic movements. F. exp. Biol. 49, 223-50.

DijkgRAAF, S. (1956). Kompensatorische Augenstieldrehungen und ihre Auslösung bei der Languste (Palinurus vulgaris). Z. vergl. Physiol. 38, 491-520.

Parker, T. J. \& Rich, J. G. (1893). Observations on the myology of Palinurus Edwardsii, Hutton. MacLeay Mem. Vol., Linn. Soc. New So. Wales, Sydney. pp. 159-78.

SCHÖNE, H. \& SCHÖNE, H. (1967). Integrated function of statocyst and antennular proprioceptive organ in the spiny lobster. Naturwiss. 54, 289.

WIERSMA, C. A. G. (1958). On the functional connections of single units in the central nervous system of the crayfish, Procambarus clarkii Girard. F. Comp. Neurol. 110, 421-71.

Wiersma, C. A. G. \& OberJat, T. (1968). The selective responsiveness of various crayfish oculomotor fibers to sensory stimuli. Comp. Biochem. Physiol. 26, 1-16.

WIERSMA, C. A. G. \& FIORE, L. (1971 $a$ ). Factors regulating the discharge frequency in optomotor fibres of Carcinus maenas. F. exp. Biol. 54, 497-505.

WiERSMA, C. A. G. \& FIORE, L. (1971 $b$ ). Unidirectional rotation neurones in the optomotor system of the crab, Carcinus. F. exp. Biol. 54, 507-1 3.

Wiersma, C. A. G. \& Yamaguchi, T. (1967). The integration of visual stimuli in the rock lobster. Vision Res. 7, 197-204.

WiERSMA, C. A. G. \& YoRK, B. (1972). Properties of the seeing fibers in the rock lobster: field structure, habituation, attention and distraction. Vision Res. 12, 627-40.

York, B., Wiersma, C. A. G. \& Yanagisawa, K. (1972). Properties of the optokinetic motor fibres in the rock lobster: build-up, flipback, afterdischarge and memory, shown by their firing patterns. F. $\exp$. Biol. 57, 217-27.

\section{EXPLANATION OF PLATE}

Fig. I. Statocyst input on to a tonic eye-down fibre with the animal in a $45^{\circ}$ eye-down position. Downward arrows: antennules are rotated along their length axis, bringing the statocyst openings toward the eyecup (excitatory). Upward arrows: antennules are rotated along their long axes, moving the statocyst openings away from the eyecup (inhibitory). Time: 5 sec.

Fig. 2. Ipsilateral antennal input on to a tonic eye-down fibre. First and second downward arrows: sudden turns of the antennal base forward and flagellum downward. First upward arrow: gradual turn of antennal base backward and flagellum upward. Second upward arrow: sudden turn of antennal base backward and flagellum upward (followed by an unmarked sudden turn forward and downward). Time: $5 \mathrm{sec}$.

Fig. 3. Input from the leg joints (mainly the basi-ischio) on to a tonic eye-down fibre. Animal in normal position. Note that in (a) leg flexion excites, whereas in (b) flexion inhibits; leg extension, on the other hand, excites in $(b)$ and inhibits in $(a)$.

(a) Manipulation of ipsilateral legs. (b) Manipulation of contralateral legs.

Upward arrows: all legs are lifted into a flexed position. Downward arrows: all legs are extended. Time: 5 sec.

Fig. 4. Effect of shadowing the ipsilateral eye on the firing pattern of a tonic head-down fibre. Note that the rather regular rest discharge becomes a bursting type during shadowing, each burst being synchronized with the shadowing speed. Time: $\frac{1}{2}$ and $\mathrm{I}$ sec.

Fig. 5. Inhibition of the discharge of a tonic eye-withdrawal fibre by the excited state brought about by mechanical stimulation. Upward arrows: commencement of mechanical stimulation. Downward arrows: end of mechanical stimulation. Time: $\frac{1}{2}$ and I sec. 

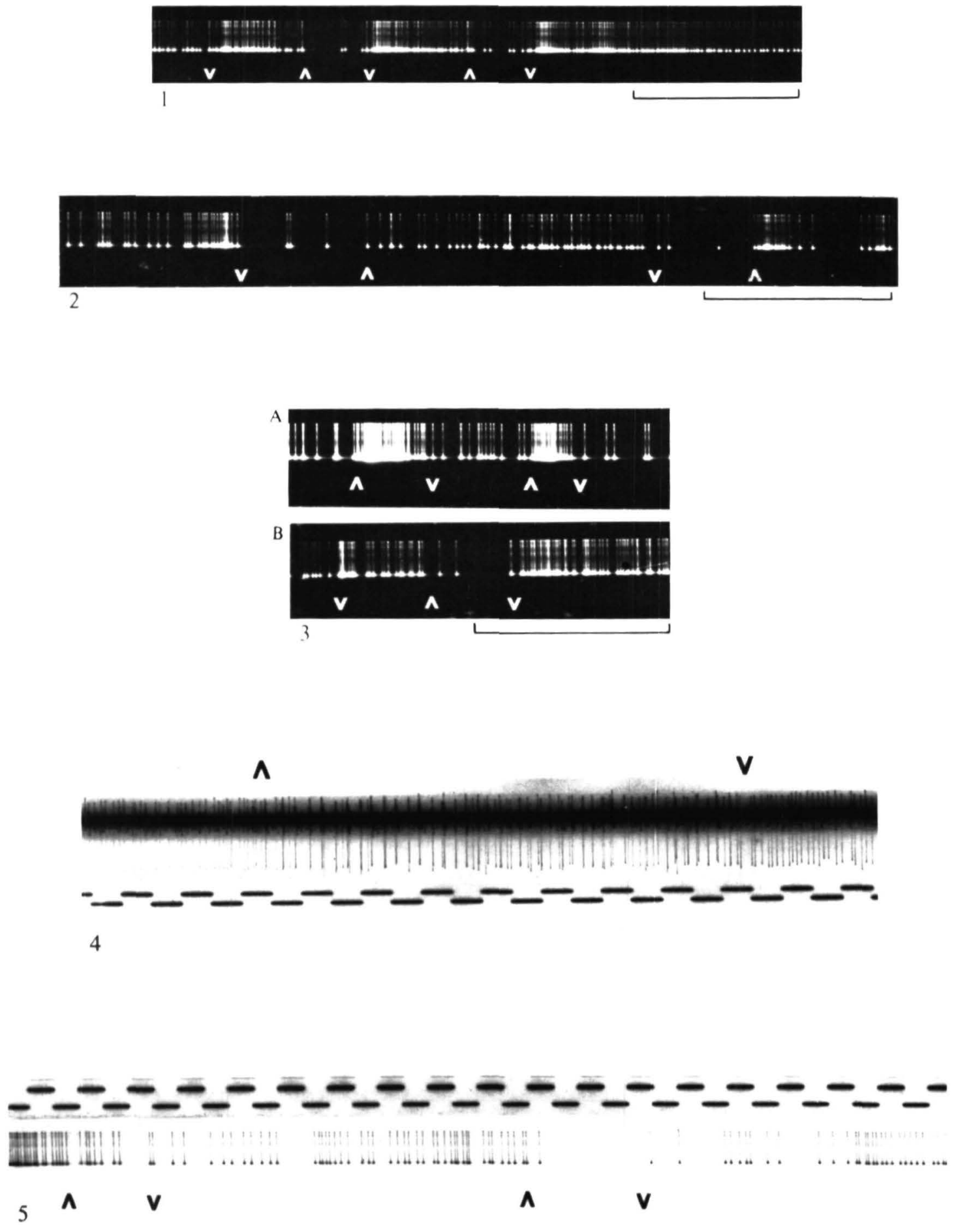

B. YORK, K. YANAGISIIWA ANI ('. A. (;. WIERSMA 
